\title{
The Research of Fretting Fatigue Characteristics of Titanium Alloys
}

\author{
Zhanjun Liu \\ Faculty of Aerospace Engineering,Shenyang Aerospace University,Shenyang 110136,China \\ luzluza@163.com
}

Keywords: fretting fatigue; wear; high cycle fatigue; titanium alloy;

\begin{abstract}
Modern aeroengine compressor and plate are made of titanium alloy, disc contacting exists between normal stress and the relative sliding value slightly. The combination of mechanical and chemical produced, including fretting fatigue and fretting wear of micro damage. Micro cracks of fretting fatigue and fretting wear size cause the platters, which is loss of normal coordinate relations between micro wear, it is caused by corrosion damage of table picture, to reduce the fatigue resistance of the parts. Titanium alloy compressor blade and micro damage parts of the disc are in the disc surface contact, breaking down is very difficult to test or monitor. In addition, so far no system can be effective to predict and prevent the micro damage in the design stage.
\end{abstract}

\section{Introduction}

High cycle fatigue failure is almost involved in each of the titanium alloy parts, such as engine compressor blades, compressor internal ring and casing, etc. High cycle fatigue damage refers to material far small fatigue fracture under the yield stress, the fatigue life is in commonly $10^{5}$ weeks time. Titanium alloy high cycle fatigue is one of the typical characteristics of the fatigue performance, which is sensitive to tissue types and surface state, the tissue types for the effect on the fatigue performance has the introduction, the titanium alloy parts surface integrity effects on fatigue characteristics[1] [4].

Titanium alloy high cycle fatigue fracture is the most important characteristics of fatigue, notch sensitivity is high, that is the defect sensitivity. Fatigue notch sensitivity is to assess materials under cyclic loading on the sensitive degree of the gap. Titanium alloy is at the same time the high cycle fatigue fracture, the extreme sensitivity of surface defect can be also surface roughness with fatigue specimens, size and dispersity have direct evidence of the fatigue life.

The surface roughness of different surface of titanium alloy has enormous influence on the fatigue limit, such as using the same process, it decreases the surface roughness of the titaniumalloy, the fatigue limit is increased from $320 \mathrm{mpa}$ to $480 \mathrm{mpa}$, the rate is as high as $50 \%$. The factors is influencing fatigue strength, limit of specimen diameter increased level drawdown, especially titanium alloy, such as is the same state of TC6 alloy, when sample is from $10 \mathrm{~mm}$ to $5 \mathrm{~mm}$ in diameter. The fatigue limit is by $15 \%$. The influence also got similar results in the alloy BT9.

\section{The research of high cycle fatigue characteristics of titanium alloy}

Fatigue life of titanium alloy is large dispersion, large dispersion is not only appear in close to the fatigue limit under the action of alternating stress, but also appeared in the far more than fatigue limit under the action of alternating stress. Such as TC6 alloy is in 700 mpa stress levels under rotating bending fatigue test, the test cycle times of fracture is one of the most important content. On macro fatigue, source area is including the first stage of crack initiation and extension area. Fatigue source area is located in the surface of the parts of the surface stress, because fatigue source longest is exposed to air, the medium and the crack propagation rate are slower, extrusion and wear are after repeated opening and closing, at the same time, which meets at different height in the initial crack extension, to form the radial steps or stripes. So the fatigue source area of the macroscopic characteristics is as follows, the oxidation or corrosion is heavier or darker, fracture surface 
is flat, smooth, fine, some fracture is visible flash small plane, there are outward radiation of radioactive level and radial stripes, the source region can't be seen to the fatigue curve, but it looked like the center of the launch fatigue curve outward. Fatigue fracture zone is the most typical macro feature fatigue curve, which is to identify and determine the important basis of fatigue failure. Fig. 1 is curve of limit concentration factor relationship[5].

When the fatigue crack is propagation, the instability of fast fracture occurred after the critical size, the area is called the transient fault. Size is not only depends on the size of the load, the transient breaking depends on the nature of the material.

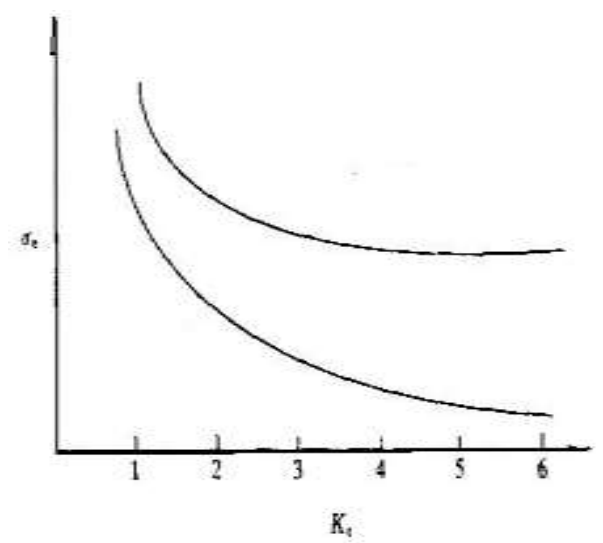

Figure 1. Curve of limit concentration factor relationship

\section{The research of fretting fatigue characteristics of titanium alloys}

Modern aeroengine compressor and plate are made of titanium alloy, disc contacting exists between normal stress and the relative sliding value slightly. The combination of mechanical and chemical produced, including fretting fatigue and fretting wear of micro damage. Micro cracks of fretting fatigue and fretting wear size cause the platters, which is loss of normal coordinate relations between micro wear, it is caused by corrosion damage of table picture, to reduce the fatigue resistance of the parts. Titanium, titanium alloy compressor blade and micro damage parts of the disc are in the disc surface contact, breaking down is very difficult to test or monitor. In addition, so far no system can be effective to predict and prevent the micro damage in the design stage. Combined with the thermal conductivity of titanium alloy is poor, friction coefficient is large, wear resistance is poorer, micro damage is very sensitive. So, the problem of micro damage between titanium alloy is sheet straight universal attention by engineering, which is also the focus of current research at home and abroad and its preventive measures. For the rotor, the micro damage is caused by fatigue fracture failure following two cases[6].

To compensate is for the expansion from high temperature, reduce its thermal stress. With the increase of rotational speed, the large centrifugal force role is in connecting the blade with the disk surface, to form large compressive stress in both close contact. At the same time, the blade is due to the effect of disturbance and vibration of air, which makes small relative sliding between them, thus appeared the kinetic friction.

They are the main factors influencing the friction behavior that contact surface, size and distribution of the stress state, micro displacement amplitude, frequency and the surface roughness and friction coefficient of the material Sometimes the cause of blade micro fatigue damage is only contact state changed, and the other conditions have not changed. If some type of titanium alloy is used manufacturing engine compressor blade, the contact surface is plated silver. No damage occurs after the operation, after changing for film coating, only $89 \mathrm{~h}$ operation is in severe fatigue damage, and led to the blade fatigue fracture failure. When channel interface is too tight, it will make its compressive stress friction, and the body boundary tangential stress increases accordingly, at the same time the lubrication effect is of dry film coating as silver coating, friction is also increasing, led to increasing damage, to reduce the fatigue life of blade. 
Under a cyclic load, the connecting contact badge is dynamic damage on the surface of the maximum of comprehensive parameters, and the corresponding fatigue life. The aircraft engine blade and wheel exists in the process of working temperature cycle, the wheeljoint can't take the solid loading, which can be made to reduce and prevent the micro. The connection interface between the relative sliding is inevitable. In this case, in order to prevent or reduce between the two arms of dynamic damage, it can adopt the following measures:

It reasonably selects vice material, try to make the blade with the linear expansion coefficient, wheel material close to choose the material with low expansion coefficient, on the surface of the micro, residual compressive stress adopts measures such as shot peening and cold rolling, silver is plated on the surface of the micro contact surface coating.

\section{The environment on the influence of titanium alloy fatigue damage}

Mentioned before, and titanium alloy is with high specific strength and show that most of the state of titaniumalloy is in the low cycle fatigue process, which usually assumes the cyclic softening, therefore the use of titanium alloy is in aviation parts and components, it is far less than the yield strength of material itself, the power of the fatigue damage is of the vast majority of consumption in the expansion of the crack initiation and crack near the threshold value, with the influence of the environment, the fatigue damage is mainly considered in this stage. Actually most of the studies show that environment is on the titanium alloy fatigue crack extension stage, stable effect on the fatigue crack propagation is also smaller in the second stage, especially under the condition of alternating, frequency is higher. They can clear that when the cyclic time, different gas pressure has no effect on the rate of crack propagation, and under the cyclic time, the influence of the environment is quite obvious.

Environmental impact is less reported on thorium alloy fatigue fracture characteristic. In general, especially the corrosive medium in environment often leads to fracture, which does not occur under the condition of using metal materials cleavage fracture. Such as titanium alloy is in the medium containing chloride.

\section{The fatigue fracture failure prevention of titanium alloy}

Although there are many factors influencing the titanium alloy parts of fatigue damage, preventive measures are mainly from the design and manufacture, the improving the fatigue resistance is of materials or components. Due to the complexity of manufacturing conditions, using of the diversity and aviation equipment is set as one of the modern high products. Its development will inevitably restricted by scientific and technological level. So sometimes design thoughtless, analysis and calculation are not accurated, which makes the parts under load far geting component itself with the resistance, the fatigue failure parts is other forms of destruction.

\section{The stress distribution pay special attention to the geometry of complex parts of the analysis}

Surface integrity includes not only the surface rough skin, the density of surface protective layer, and the outside factors that is caused by mechanical damage, and including the surface residual stress of type, size and distribution. Because titanium alloy has low thermal conductivity, and the coefficient of friction during processing is prone to large, the uneven distribution is of residual tensile stress in developed countries, the third was adopted to realize synchronous residual stress measurement in the machining process, the method is to use the real-time detection of X-ray stress analysis, the residual stress analysis results timely feedback, processing parameters, which is to adjust the process to make the surface of the component complete guaranteed.

Manufacturing process want to strictly abide by the norms, and avoid quality that does not meet the requirements of the situation, especially to prevent parts of stress concentration. Fatigue fracture failure mostly is originated in the components of the angle, chamfering, slotting and the arc transitional area and so on, especially for the titanium alloy fatigue notch sensitivity. The surface layer of shot peening makes material 
produce the following two major changes, residual compressive stress field is introduced, Cyclic plastic deformation leads to organization structure change of surface materials. Smooth surface of the parts to the actual parts is that the results of surface strengthening is the original table of fatigue crack initiation in parts driven skin surface initiation, which is residual compressive stress that is introduced into the main role in the surface layer.

\section{Surface shot peening treatment can greatly improve the fatigue strength of titanium alloy}

This is because the cutting of titanium alloy poor processability, stick knife phenomenon is easy to cause high alloy surface roughness, surface residual stress is caused by uneven distribution, which even is likely to produce certain tensile stress, and shot peening can not only make the surface form a uniform distribution of residual compressive stress, and to a certain extent, reduce the machining defects, to improve the surface integrity. Titanium alloy is given to use the fatigue limit of different process parameters before and after shot peening. That shot peening raises the three point bending of fatigue strength limit of sheet. For the processing quality well, the shot peening is compressive stress of TC 9 thorium alloy, the fatigue limit is increased by $26 \%$. Seen at the same time, the spray is both residual compressive stress and the residual tensile stress, shot peening is for uniform distribution of residual compressive stress.

Residual stress has two characteristics of introduction of blade surface by machining, the whole stress distribution uniformity is poor, and sometimes in some place there may be tension stress, the residual stress is in the shallow depth, usually less than $0.05 \mathrm{~mm}$. And it is introduced through shot peening residual stress, the blade has the following characteristics, stress distribution is on the whole piece of uniform, residual stress is produced by spray layer, its depth can reach $0.3 \mathrm{~mm}$ to $0.35 \mathrm{~mm}$, it is on fatigue crack surface in depth, times is the role of the surface of initiation, thus which improves the fatigue strength.

\section{Conclusions}

Modern aeroengine compressor and plate are made of titanium alloy, disc contacting exists between normal stress and the relative sliding value slightly. The combination of mechanical and chemical produced, including fretting fatigue and fretting wear of micro damage. Micro cracks of fretting fatigue and fretting wear size cause the platters, which is loss of normal coordinate relations between micro wear, it is caused by corrosion damage of table picture, to reduce the fatigue resistance of the parts. Titanium alloy compressor blade and micro damage parts of the disc are in the disc surface contact, breaking down is very difficult to test or monitor. In addition, so far no system can be effective to predict and prevent the micro damage in the design stage.

\section{References}

[1] Montmionnet P, Delamare F. Wear, (2000), p125 135.

[2] Tabary and Deneuville,P. ASME J.Tribology ,(1996),p629 636.

[3] Roberts, W.L.Cold rolling of steel ,(1988).

[4] Rowe,C.N. ASLE Trans.(1996), p101 111.

[5]Feng. Jing, The Mixture of The Multimode System Reliability Growth Model, Computer Application Research Supplement, pp.60-61, 2003.

[6] Xu. Yuxiu, Complex Mechanical Fault Diagnosis Method of Fractal and Wavelet, Mechanical Industry Publishing House,Beijing, pp.24-25,2005. 